\title{
An Alternative Single Parameter Functional Form for Lorenz Curve
}

\section{Crawford School Working Paper 1712 September 2017}

\section{Satya Paul}

Amrita University, India

\section{Sriram Shankar}

Australian National University

\section{Abstract}

This paper proposes a single parameter functional form for the Lorenz curve and compares its performance with the existing single parameter functional forms using Australian income data for 10 years. The proposed parametric functional form performs better than the existing Lorenz functional forms. The Gini based on the proposed functional form is closest to true Gini each year.

Keywords: Gini coefficient; Lorenz curve; Parametric functional form JEL Classification: C80, D31 


\section{Suggested Citation:}

Paul, S and Shankar, S. (2017) An Alternative Single Parameter Functional Form for Lorenz Curve, Crawford School working paper 1712, September 2017. Crawford School of Public Policy, The Australian National University.

Address for Correspondence:

Name: Sriram Shankar

Position: Fellow

Address: 2.21 Beryl Rawson Building, ANU Centre for Social Research and Methods, Australian National University

Email: Sriram.Shankar@anu.edu.au

Crawford School of Public Policy

College of Asia and the Pacific

The Australian National University

Canberra ACT 0200 Australia

www.anu.edu.au

The Crawford School of Public Policy is the Australian National University's public policy school, serving and influencing Australia, Asia and the Pacific through advanced policy research, graduate and executive education, and policy impact. 
An Alternative Single Parameter Functional Form for Lorenz Curve

\author{
Satya Paul \\ Amrita University, India \\ and \\ Sriram Shankar ${ }^{1}$ \\ Australian National University
}

\begin{abstract}
This paper proposes a single parameter functional form for the Lorenz curve and compares its performance with the existing single parameter functional forms using Australian income data for 10 years. The proposed parametric functional form performs better than the existing Lorenz functional forms. The Gini based on the proposed functional form is closest to true Gini each year.
\end{abstract}

Keywords: Gini coefficient; Lorenz curve; Parametric functional form JEL Classification: C80, D31

\footnotetext{
${ }^{1}$ Corresponding author: 2.21 Beryl Rawson Building, ANU Centre for Social Research and Methods, Australian National University, email: Sriram.Shankar@anu.edu.au. Contact details for Satya Paul are: Centre for Economics and Governance, Amrita University, Kerala, India. Email: satyapaul@outlook.com.
} 


\section{Introduction}

The Lorenz curve represents a graphical relationship between the cumulative proportion of population and the cumulative proportion of income and forms the backbone of several inequality measures including the widely used Gini coefficient. World Institute of Development Economics Research (WIDER) and World Bank publish data on income shares by decile or quintile groups of population for a large number of countries. Based on group data, Lorenz curve can be constructed (i) by interpolation techniques (Gastwirth and Glauberman, 1976), (ii) by assuming a statistical distribution of income and deriving the Lorenz curve (McDonald, 1984), or (iii) by specifying a parametric functional form for the Lorenz curve. The interpolation techniques assume the homogeneity of incomes within sub-groups, thereby leading to a downward bias in Gini estimate. The existing income distribution functions are known to be poorly fitting the Lorenz curve and resulting in inaccurate inequality estimates ${ }^{2}$. The parametric Lorenz functional forms are directly estimated with the group data without assuming homogeneity of incomes within sub-groups and thus are not downwardly biased. Various authors have suggested a variety of parametric functional forms to directly estimate Lorenz curve (Kakwani and Podder, 1973; Aggarwal, 1984; Gupta, 1984; Chotikapanich, 1993; Rohde, 2009). It would be useful and interesting to determine which functional form performs best. This is particularly important when the aim is to construct inequality measures based on Lorenz functional form.

This paper proposes an alternative single parameter Lorenz functional form and compares its performance with the existing single parametric functional forms. The Australian data are used to compare the goodness-of-fit of the widely used parametric Lorenz specifications and the one suggested here

\section{Alternative Functional Forms for the Lorenz Curve}

If $\mathrm{p}(\mathrm{x})$ is the proportion of individuals that receive an income up to $\mathrm{x}$ and $\eta$ is the proportion of total income received by the same units, then the Lorenz curve is defined as

$$
\eta=\mathrm{L}(\mathrm{p}(\mathrm{x}))
$$

The regularity conditions for the function L(p) to describe the Lorenz curve are as follows.

$$
\text { (i) } \mathrm{L}(0)=0 \text {, (ii) } \mathrm{L}(1)=1 \text {, (iii) } \frac{\mathrm{dL}}{\mathrm{dp}} \geq 0 \text { and (iv) } \frac{\mathrm{d}^{2} \mathrm{~L}}{\mathrm{dp}^{2}}>0 \text {. }
$$

\footnotetext{
${ }^{2}$ Also see Chotikapanich (1993).
} 
Note that (i) and (ii) imply that the Lorenz curve is defined over the domain $0 \leq \mathrm{p} \leq 1$ and (iii) and (iv) suggest that the slope of Lorenz curve is non-negative and monotonically increasing. We propose the following functional form for the Lorenz curve

$$
L(p)=p\left[\frac{e^{-\gamma\left(1-e^{p}\right)}-1}{e^{-\gamma(1-e)}-1}\right] \text {, where } \gamma>0
$$

It is easy to verify that Eq. (2) passes through the coordinate points $(0,0)$ and $(1,1)$ and that the first and second derivatives are greater than zero. That is, $L(0)=0, L(1)=1$ and

$$
\begin{aligned}
& L^{\prime}(p)=\left[\frac{e^{-\gamma\left(1-e^{p}\right)}-1}{e^{-\gamma(1-e)}-1}\right]+p \gamma \frac{e^{-\gamma\left(1-e^{p}\right)+p}}{e^{-\gamma(1-e)}-1} \geq 0 \\
& L^{\prime \prime}(p)=2 \gamma \frac{e^{-\gamma\left(1-e^{p}\right)+p}}{e^{-\gamma(1-e)}-1}+p \gamma\left(\gamma e^{p}+1\right) \frac{e^{-\gamma\left(1-e^{p}\right)+p}}{e^{-\gamma(1-e)}-1}>0 \text { for } \gamma>0,0 \leq p \leq 1
\end{aligned}
$$

This functional form is compared with the existing widely used single parameter functional forms proposed by Kakwani and Podder (1973), Chotikapanich (1993) and Rohde (2009) ${ }^{3}$ and a form implied by Pareto distribution.

$$
\begin{array}{ll}
\text { Pareto: } & L(p)=1-(1-p)^{\frac{1}{\alpha}}, \alpha>1 \\
\text { Kakwani-Podder: } & L(p)=p e^{-\delta(1-p)}, \delta>0 \\
\text { Chotikapanich: } & L(p)=\frac{e^{\kappa p}-1}{e^{\kappa}-1}, \kappa>0 \\
\text { Rohde: } & L(p)=p\left(\frac{\beta-1}{\beta-p}\right), \beta>1
\end{array}
$$

The main motivation for fitting a Lorenz curve is to facilitate the estimation of inequality measures such as the Gini coefficient. This widely used index is defined as one minus twice the area under the Lorenz curve. Based on the proposed (new) and existing functional forms, Gini coefficients are expressed as follows:

\footnotetext{
${ }^{3}$ We do not consider here two other single parameter functional forms given by Aggrawal (1984) and Gupta (1984). As pointed out in Sarabia et al (2010), their functional forms do not add substantive value to the Lorenz curve comparison, as Rohde (2009) is a reparameterization of Aggarwal (1984), and Gupta (1984) that of Kakwani and Podder (1973).
} 
$\begin{array}{ll}\text { Proposed: } & G=1-2 \int_{0}^{1} p\left[\frac{e^{-\gamma\left(1-e^{p}\right)}-1}{e^{-\gamma(1-e)}-1}\right] d p \\ \text { Pareto: } & G=1-2 \int_{0}^{1}\left[1-(1-p)^{\frac{1}{\alpha}}\right] d p \\ \text { Kakwani-Podder: } & G=1-2 \int_{0}^{1} p e^{-\delta(1-p)} d p \\ \text { Chotikapanich: } & G=\frac{(\kappa-2) \mathrm{e}^{\kappa}+(\kappa+2)}{\kappa\left(\mathrm{e}^{\kappa}-1\right)} \\ \text { Rohde: } & G=2 \beta\left[(\beta-1) \ln \left(\frac{\beta-1}{\beta}\right)+1\right]-1\end{array}$

\section{Performance of Alternative Functional Forms of Lorenz Curve}

Table 1 presents the Australian income data by decile groups for 2001-20104. Based on these data, the proposed and other four functional forms of Lorenz curve (equations 2 and 5 to 8) are estimated using the non-linear least squares. All the parameter estimates are statistically significant at the 1\% level (Table 2). A comparison of these estimated functional forms is done based on two statistics.

(i) Information Inaccuracy Measure (I) $=\sum_{\mathrm{i}=1}^{\mathrm{N}} \mathrm{q}_{\mathrm{i}} \ln \left(\mathrm{q}_{\mathrm{i}} / \hat{\mathrm{q}}_{\mathrm{i}}\right)$

where $\mathrm{q}_{\mathrm{i}}$ and $\hat{\mathrm{q}}_{\mathrm{i}}$ denote actual and predicted income shares. The estimated function with smaller value of $I$ is better than those with larger values.

(ii) Mean Square Error $(\mathrm{MSE})=\frac{1}{\mathrm{~N}} \sum_{\mathrm{i}=1}^{\mathrm{N}}\left[\eta_{\mathrm{i}}-\mathrm{L}\left(\mathrm{p}_{\mathrm{i}}, \hat{\theta}\right)\right]^{2}$.

It is always non-negative, and values closer to zero are better. Both the statistics are measures of goodness-of-fit.

\footnotetext{
${ }^{4}$ These group data were constructed using the individual income data from the first ten waves (2001-2010) of Household, Income and Labour Dynamics Australia (HILDA) Survey.
} 
In terms of measure $I$, the proposed functional form performs best followed by KakwaniPodder, Chotikapanich, Rohde and Pareto respectively in each year (Table 3). In terms of MSE, the proposed functional form performs best in seven years, 2001 to 2006 and 2009, whereas Kakwani-Podder functional form performs best in the other 3 years (Table 4). In 2007 and 2010, the Chotikapanich functional form performs the second best. The worst performance is shown again by the Pareto functional form.

Table 1: Actual Income Shares by Decide Groups, 2001-2010

\begin{tabular}{|c|c|c|c|c|c|c|c|c|c|c|}
\hline $\begin{array}{c}\text { Decide } \\
\text { Groups }\end{array}$ & 2001 & 2002 & 2003 & 2004 & 2005 & 2006 & 2007 & 2008 & 2009 & 2010 \\
\hline 1 & 0.0009 & 0.0009 & 0.0010 & 0.0012 & 0.0013 & 0.0012 & 0.0009 & 0.0011 & 0.0011 & 0.0009 \\
\hline 2 & 0.0146 & 0.0145 & 0.0135 & 0.0166 & 0.0186 & 0.0193 & 0.0161 & 0.0168 & 0.0164 & 0.0170 \\
\hline 3 & 0.0364 & 0.0364 & 0.0365 & 0.0386 & 0.0401 & 0.0400 & 0.0389 & 0.0389 & 0.0395 & 0.0387 \\
\hline 4 & 0.0494 & 0.0505 & 0.0526 & 0.0544 & 0.0547 & 0.0547 & 0.0526 & 0.0528 & 0.0545 & 0.0533 \\
\hline 5 & 0.0705 & 0.0713 & 0.0733 & 0.0736 & 0.0738 & 0.0746 & 0.0727 & 0.0733 & 0.0765 & 0.0714 \\
\hline 6 & 0.0944 & 0.0947 & 0.0952 & 0.0956 & 0.0951 & 0.0946 & 0.0932 & 0.0949 & 0.0960 & 0.0943 \\
\hline 7 & 0.1176 & 0.1177 & 0.1179 & 0.1178 & 0.1155 & 0.1149 & 0.1126 & 0.1147 & 0.1174 & 0.1138 \\
\hline 8 & 0.1443 & 0.1440 & 0.1450 & 0.1432 & 0.1409 & 0.1393 & 0.1366 & 0.1389 & 0.1406 & 0.1384 \\
\hline 9 & 0.1804 & 0.1804 & 0.1793 & 0.1782 & 0.1759 & 0.1744 & 0.1717 & 0.1737 & 0.1738 & 0.1729 \\
\hline 10 & 0.2915 & 0.2896 & 0.2857 & 0.2808 & 0.2841 & 0.2870 & 0.3047 & 0.2949 & 0.2842 & 0.2993 \\
\hline
\end{tabular}

Source: These decide groups are constructed using the data on individual income from first 10 waves of HILDA Survey.

Table 2: Estimates of Lorenz Parameters (2001-2010)

\begin{tabular}{cccccccccccc}
\hline & \multicolumn{2}{c}{ Proposed } & \multicolumn{2}{c}{ Rohde } & \multicolumn{3}{c}{ Chotikapanich } & \multicolumn{3}{c}{ Pareto } & \multicolumn{3}{c}{ Kakwani-Podder } \\
\hline Year & $\gamma$ & SE & $\beta$ & SE & $\kappa$ & SE & $\alpha$ & SE & $\delta$ & SE \\
\hline 2001 & 0.259 & 0.040 & 1.327 & 0.026 & 3.096 & 0.080 & 2.494 & 0.238 & 2.137 & 0.059 \\
2002 & 0.244 & 0.040 & 1.331 & 0.027 & 3.074 & 0.081 & 2.480 & 0.236 & 2.119 & 0.059 \\
2003 & 0.213 & 0.040 & 1.338 & 0.028 & 3.033 & 0.085 & 2.452 & 0.234 & 2.084 & 0.062 \\
2004 & 0.154 & 0.042 & 1.353 & 0.028 & 2.947 & 0.080 & 2.398 & 0.220 & 2.010 & 0.058 \\
2005 & 0.142 & 0.049 & 1.356 & 0.027 & 2.925 & 0.077 & 2.390 & 0.210 & 1.992 & 0.057 \\
2006 & 0.144 & 0.053 & 1.355 & 0.026 & 2.927 & 0.078 & 2.393 & 0.207 & 1.993 & 0.059 \\
2007 & 0.248 & 0.062 & 1.329 & 0.023 & 3.069 & 0.086 & 2.492 & 0.217 & 2.114 & 0.071 \\
2008 & 0.203 & 0.054 & 1.340 & 0.025 & 3.010 & 0.082 & 2.448 & 0.217 & 2.063 & 0.064 \\
2009 & 0.139 & 0.049 & 1.356 & 0.028 & 2.925 & 0.084 & 2.389 & 0.215 & 1.992 & 0.063 \\
2010 & 0.226 & 0.057 & 1.335 & 0.024 & 3.040 & 0.082 & 2.469 & 0.217 & 2.088 & 0.066
\end{tabular}


Table 3 Information Inaccuracy Measure

\begin{tabular}{cccccc}
\hline Year & Proposed & Rohde & Chotikapanich & Pareto & Kakwani-Podder \\
\hline 2001 & 0.0073 & 0.0373 & 0.0205 & 0.1070 & 0.0172 \\
2002 & 0.0073 & 0.0378 & 0.0209 & 0.1072 & 0.0175 \\
2003 & 0.0074 & 0.0394 & 0.0221 & 0.1087 & 0.0185 \\
2004 & 0.0073 & 0.0367 & 0.0211 & 0.1022 & 0.0178 \\
2005 & 0.0085 & 0.0344 & 0.0209 & 0.0955 & 0.0180 \\
2006 & 0.0096 & 0.0342 & 0.0215 & 0.0932 & 0.0187 \\
2007 & 0.0125 & 0.0355 & 0.0236 & 0.0927 & 0.0211 \\
2008 & 0.0102 & 0.0354 & 0.0221 & 0.0954 & 0.0193 \\
2009 & 0.0092 & 0.0378 & 0.0232 & 0.0994 & 0.0200 \\
2010 & 0.0112 & 0.0352 & 0.0226 & 0.0942 & 0.0200
\end{tabular}

Table 4 Mean Squared Errors (MSE)

\begin{tabular}{cccccc}
\hline Year & Proposed & Rohde & Chotikapanich & Pareto & Kakwani-Podder \\
\hline 2001 & 0.00012 & 0.00084 & 0.00023 & 0.00409 & 0.00017 \\
2002 & 0.00012 & 0.00085 & 0.00023 & 0.00409 & 0.00017 \\
2003 & 0.00012 & 0.00090 & 0.00026 & 0.00416 & 0.00019 \\
2004 & 0.00014 & 0.00081 & 0.00024 & 0.00390 & 0.00018 \\
2005 & 0.00019 & 0.00070 & 0.00022 & 0.00360 & 0.00018 \\
2006 & 0.00022 & 0.00067 & 0.00023 & 0.00349 & 0.00019 \\
2007 & 0.00030 & 0.00065 & 0.00026 & 0.00340 & 0.00025 \\
2008 & 0.00023 & 0.00070 & 0.00025 & 0.00357 & 0.00021 \\
2009 & 0.00018 & 0.00080 & 0.00027 & 0.00376 & 0.00021 \\
2010 & 0.00025 & 0.00067 & 0.00024 & 0.00349 & 0.00022
\end{tabular}

The true Ginis and the estimated Ginis based on alternative functional forms of Lorenz curve are statistically significant at the $1 \%$ level. (Table 5). For each year, the Gini based on the proposed Lorenz curve specification is closest to true Gini. The Gini based on the KakwaniPodder functional form is second closest to true Gini. The Ginis based on the Chotikapanich, Rohde and Pareto Lorenz curve functional forms rank respectively third, fourth and fifth in terms of their closeness to true Gini. 
Table 5: Estimates of Gini (2001-2010)

\begin{tabular}{clllllllllllll}
\hline & & & & & \multicolumn{4}{c}{ Chotika- } & \multicolumn{4}{c}{ Kakwani- $^{2}$} \\
Year & TRUE & SE & Proposed & SE & Rohde & SE & panich & SE & Pareto & SE & Podder & SE \\
\hline 2001 & 0.4633 & 0.0024 & 0.4576 & 0.0061 & 0.4384 & 0.0084 & 0.4487 & 0.0034 & 0.4276 & 0.0390 & 0.4504 & 0.0075 \\
2002 & 0.4613 & 0.0025 & 0.4553 & 0.0061 & 0.4361 & 0.0084 & 0.4464 & 0.0035 & 0.4253 & 0.0390 & 0.4481 & 0.0077 \\
2003 & 0.4572 & 0.0026 & 0.4506 & 0.0060 & 0.4316 & 0.0087 & 0.4418 & 0.0037 & 0.4206 & 0.0393 & 0.4435 & 0.0081 \\
2004 & 0.4464 & 0.0026 & 0.4416 & 0.0064 & 0.4226 & 0.0082 & 0.4321 & 0.0035 & 0.4115 & 0.0381 & 0.4337 & 0.0078 \\
2005 & 0.4442 & 0.0025 & 0.4397 & 0.0075 & 0.4208 & 0.0077 & 0.4297 & 0.0033 & 0.4100 & 0.0366 & 0.4313 & 0.0078 \\
2006 & 0.4446 & 0.0025 & 0.4400 & 0.0081 & 0.4212 & 0.0075 & 0.4298 & 0.0034 & 0.4106 & 0.0360 & 0.4314 & 0.0081 \\
2007 & 0.4619 & 0.0025 & 0.4559 & 0.0094 & 0.4371 & 0.0074 & 0.4458 & 0.0037 & 0.4272 & 0.0356 & 0.4474 & 0.0091 \\
2008 & 0.4547 & 0.0026 & 0.4491 & 0.0083 & 0.4303 & 0.0077 & 0.4392 & 0.0036 & 0.4199 & 0.0365 & 0.4408 & 0.0084 \\
2009 & 0.4455 & 0.0025 & 0.4393 & 0.0074 & 0.4206 & 0.0082 & 0.4297 & 0.0037 & 0.4099 & 0.0374 & 0.4313 & 0.0085 \\
2010 & 0.4579 & 0.0025 & 0.4525 & 0.0087 & 0.4337 & 0.0075 & 0.4425 & 0.0036 & 0.4235 & 0.0360 & 0.4441 & 0.0086
\end{tabular}

Note: True Ginis are calculated using the data on individual income from the first 10 waves of HILDA Survey. Standard errors for true Ginis are calculated based on a method provided in Giles (2004). The expressions for the variances for Ginis corresponding to the alternative parametric functional forms of Lorenz curve are presented in Appendix A.

\section{Concluding Remarks}

The Australian data show the superiority of the proposed Lorenz curve functional form over other functional forms. In terms of information inaccuracy measure, the proposed form outperforms all the four functional forms in all the 10 years, However, in terms of MSE, the proposed Lorenz functional form performs best in 7 years whereas the Kakwani-Podder functional form performs best in the other 3. The Gini based on our Lorenz curve specification is closest to true Gini each year. 


\section{Appendix A}

The standard error for Gini coefficient is calculated using the asymptotic approximation: $\operatorname{var}(\hat{G})=\left(\frac{d G}{d \theta}\right)^{2} V_{\theta}$, where $V_{\theta}$ is the asymptotic variance of the single parameter $\theta$ estimated using non-linear least squares for the various functional forms of Lorenz curve.

Chotikapanich: $\operatorname{Var}(G)=\left[\frac{2\left(e^{\hat{\kappa}}\left(e^{2}-\hat{\kappa}^{2}-2\right)+1\right)}{\left(\hat{\kappa}\left(e^{\hat{\kappa}}-1\right)\right)^{2}}\right]^{2} \operatorname{Var}(\hat{\kappa})$.

Rohde: $\operatorname{Var}(G)=\left(\frac{d G}{d \beta}\right)^{2} \operatorname{Var}(\beta)$, where $\frac{d G}{d \beta}=2\left[(2 \beta-1) \ln \left(\frac{\beta-1}{\beta}\right)+2\right]$.

Kakwani-Podder: $\operatorname{Var}(G)=\left(\frac{d G}{d \delta}\right)^{2} \operatorname{Var}(\hat{\delta})$, where $\frac{d G}{d \delta}=2 \int_{0}^{1} p(1-p) e^{-\delta(1-p)} d p$.

Pareto: $\operatorname{Var}(G)=\left(\frac{d G}{d \alpha}\right)^{2} \operatorname{Var}(\alpha)$, where $\frac{d G}{d \alpha}=-2 \int_{0}^{1} \frac{1}{\alpha^{2}} \ln (1-p)(1-p)^{\frac{1}{\alpha}} d p$.

Proposed: $\operatorname{Var}(G)=\left(\frac{d G}{d \gamma}\right)^{2} \operatorname{Var}(\hat{\gamma})$

where

$$
\frac{d G}{d \gamma}=2 \int_{0}^{1}\left(-\frac{1-e^{p}}{1-e^{\gamma\left(1-e^{p}\right)}}+\frac{1-e}{1-e^{\gamma(1-e)}}\right)\left(p\left[\frac{e^{-\gamma\left(1-e^{p}\right)}-1}{e^{-\gamma(1-e)}-1}\right]\right) d p .
$$

\section{Acknowledgements}

This article uses unit record data from the HILDA Survey. The HILDA Project was initiated and is funded by the Australian Government Department of Families, Housing, Community Services and Indigenous Affairs (FaHCSIA) and is managed by the Melbourne Institute of Applied Economic and Social Research (Melbourne Institute). The findings and views reported in this article, however, are those of the authors and should not be attributed to either

FaHCSIA or the Melbourne Institute. 


\section{References}

Aggarwal, V., 1984. On optimum aggregation of income distribution data. Sankhya B, 46, 343-355.

Chotikapanich, D., 1993. A comparison of alternative functional forms for the Lorenz curve. Economics Letters, 41, 21-29.

Gastwirth, J., Glauberman, M., 1976. The interpolation of the Lorenz curve and Gini index from grouped data. Econometrica, 44, 479-483.

Giles, D. E. A., 2004. Calculating a standard error for the Gini coefficient: some further results. Oxford Bulletin of Economics and Statistics, 66, 425-433.

Gupta, M.R., 1984. Functional form for estimating the Lorenz curve. Econometrica, 52, 1313-1314.

Kakwani, N.C., Podder, N., 1973. On the estimation of Lorenz curves from grouped observations. International Economic Review, 14, 278-292.

McDonald, J. B., 1984. Some generalized functions for the size distribution of income. Econometrica, 52, 647-663.

Rohde, N., 2009. An alternative functional form for estimating the Lorenz curve. Economics Letters, 105, 61-63.

Sarabia, J.M., Prieto, F., Sarabia, M., 2010. Revisiting a functional form for the Lorenz curve. Economics Letters, 107, 249-252. 